\title{
Predictive Policing
}

\author{
Roman Povalej' · Dirk Volkmann ${ }^{1}$
}

Angenommen: 12. Januar 2021 / Online publiziert: 29. Januar 2021

(c) Der/die Autor(en) 2021

\section{Zusammenfassung}

Mit zunehmender Digitalisierung extendieren insbesondere die qualitativen und quantitativen Kriterien der polizeilichen Arbeit. Mathematische Algorithmen, wie Predictive Policing, halten immer mehr Einzug. Retrospektiv werden dabei eine effektivere und effizientere Ausdifferenzierung der Risikogebiete realisiert. Bei der prädiktiven Prognose werden mittels mathematischer Algorithmen Wahrscheinlichkeiten zur Ausweisung relevanter Risikogebiete bestimmt, die in Deutschland überwiegend raumbezogene, aber auch personenbezogene Verfahren implizieren. Im Rahmen einer Diskussion, z. T. am Beispiel des Phänomens Wohnungseinbruchdiebstähle, nähern wir uns inhaltlich dem Themenkomplex. Die Effizienz der fallspezifischen Datenanalyse implementiert die Registrierung immenser Datenmengen, die insbesondere durch die Qualität aber auch Quantität der relevanten Informationen eminent geprägt wird.

Das digitale Zeitalter beeinflusst immer stärker auch den polizeilichen Arbeitsalltag. So tragen bereits heute, und zukünftig noch mehr, mathematisch gesteuerte Algorithmen zur Optimierung von Analyse- und Optionsprozessen im Einsatz- und Streifendienst, im kriminalistischen bzw. kriminaltechnischen Bereich bei. Insbesondere die Visualisierung von kartografisch in Form von zwei- und dreidimensionalen georeferenzierten Karten als auch von raumbezogenen Kausalitäten, die Realisierung von Analysen, die Optimierung von Einsatzplanungen sowie Präventivmaßnahmen und nicht zuletzt das statistische Berichtwesen sollten noch explorativer in den Fokus rücken und eine zentralere Rolle bei den Polizeien einnehmen. Die Verwendung von Prognosesystemen bei der Polizei weckt nicht zuletzt die Erwartungshaltung, durch intelligentere Verknüpfung und Nutzung von Daten, Informationen und Systemen effizienter agieren bzw. operieren zu können [1].

Roman Povalej

roman.povalej@polizei.niedersachsen.de

Dirk Volkmann

dirk.volkmann@polizei.niedersachsen.de

1 Polizeiakademie Niedersachsen, Nienburg (Weser), Deutschland

\section{Begriffserörterung}

Predictive Policing bedeutet im wörtlichen Kontext übersetzt „Vorausschauende Polizeiarbeit“ und wird im deutschsprachigen Raum eher mit ,vorhersagebasierter Polizeiarbeit" gebraucht [8]. Allgemein geht es um Algorithmen, die größere Dateninformationen bzw. -mengen polizeispezifisch strukturiert analysieren, um individuelle Erscheinungsformen von Kriminalität zu prognostizieren [12, S. 2] und auf der Basis diverser kriminologischer Theorien, z. B. Broken Windows, Routine Activity Approach, Rational Choice Theory, Near Repeat Victimisation, Kontinuitäten i. S. v. kontinuierlichen Mustern, auch Musteridentifikation genannt, lokalisieren zu können [17, S. 2].

Die Analysen des Predictive Policing basieren in erster Linie auf der Generierung umfangreicher Datenmengen aus detaillierten Lagebildern, aktuellen Ereignissen [14, S. 459], allgemein zugänglichen Daten, perspektivisch auch von Sensordaten (Internet of Things, Smarthome) sowie aus kriminologischen Theorien, deren Kernaussagen in der Annahme darin fußen, dass aus den hinreichend vorliegenden Falldaten bzw. -zahlen auf eine erhöhte Wahrscheinlichkeit zukünftiger Kriminalität geschlossen werden kann, sodass sich bestimmte Kriminalitätsphänomene bzw. Delikte mit regionalem, aber auch überregionalem Bezug oder bei gleichen Opfern innerhalb einer kurzen Zeitspanne wiederholen $[17$, S. 3]. 


\section{Varianten}

Beim Predictive Policing kommen sowohl raumbezogene als auch personenbezogene Verfahren zum Einsatz. Raumbezogene Verfahren zeichnen sich durch Ausweisung prädiktiver Risikogebiete mit zeitlicher Präferenz aus. Aktuell werden bei den Polizeien in Deutschland die Prädiktionsmethoden Hot-Spot-Methode, Near-Repeat-Methode und die Methode Risk Terrain Analysis eingesetzt [9, S. $245 \mathrm{ff}$., 11, S. 13-19, $29 \mathrm{ff} ., 15$, S. $153 \mathrm{ff}$.]. Personenbezogene Verfahren orientieren sich in erster Linie auf eine/r Täter/-innen- und/oder einen opferorientierten Kriminalitätsprognose. In Deutschland werden diese algorithmischen Prädiktionsverfahren (X-Sonar, Radar-iTE) insbesondere zur Identifizierung von Radikalisierungsmustern und letztlich zur Abwehr von Terrorgefahren verwendet [8, S. 5, 9, S. 242f.].

Aktuell wird vermehrt an Modifizierungen der Prognosemodelle gearbeitet, um die Ausweisung der prognostizierten Risikogebiete zu optimieren und zu verifizieren.

\section{Datenqualität/-quantität}

In digitalisierten Prognoseprozessen, wie dem Predictive Policing, werden immense Datenmengen erhoben. Dabei kommt der Datenqualität eine herausragende Bedeutung zu. Insbesondere die Effizienz und Effektivität der Ergebnisse der Prozessmodelle werden durch einen sorgsamen qualitativen Umgang entsprechend gestaltet und beeinflusst [13]; d.h. die Prozesseffizienz und der damit verbundene Erfolg der Kriminalitätsprognose, gemessen an ihrer prädiktiven Wirksamkeit, hängt entscheidend von der Qualität der relevanten Daten bzw. Informationen hinsichtlich ihrer Vollständigkeit i. S.v. Gesamtheit, Korrektheit und Redundanz ab. Eine kontinuierliche Kontrolle der prozessrelevanten Daten mittels eines Datenmanagements trägt obligatorisch zur Vermeidung von Datenqualitätsdefiziten und letztlich auch zur Prozesssicherheit bei [7].

Inkonsistenzen von Daten, hervorgerufen durch eine fehlerhafte direkte Dateneingabe oder manuelle Datenübertragung, lassen sich manuell oder digital durch moderne Integrationstechniken bereinigen [13]. Sofern Datensätze in verschiedenen Systemen i.S.v. doppelter Datenhaltung in diversen Datensilos, z.B. Vorgangs- und Fallbearbeitungssystemen bzw. Analysesystemen, zwischengespeichert werden, dürfen sich diese nicht widersprechen [13], vor allem nicht bei der Erfassung von Primär- und Sekundärmetrik. Aus diesem Grund sollte beim Aufbau von Datenbanken, und der damit verbundenen Migration von bereits erfassten Datensätzen in ein Data-Warehouse, die Implementierung von Informationen, z. B. georeferenzierten (Primärmetrik) automatisch erfolgen, sodass fehlerhafte Adressdaten oder
Ortsangaben (Sekundärmetrik) selektiert und validiert werden können, z. B. manuell oder mittels Datenbereinigungsservice [16].

Auch die Datenquantität spielt im Prognoseprozess eine nicht unerhebliche Rolle. Erst durch quantitative Ansätze mittels mathematischer Algorithmen wird das Prognostizieren prädiktiver Ereignisse i. S. v. Kriminalitätsprognose ermöglicht; d.h. sowohl zur Überprüfung der Plausibilitäten bzw. Wirksamkeit des Algorithmus und der damit verbundenen Sicherheit der Aussagekraft als auch zum Erkennen möglicher Zusammenhänge bzw. Beziehungen zwischen Merkmalen ist die entsprechende Datenmenge als quantitatives Merkmal erforderlich.

\section{Diskussion}

Die Ursachen für den extrem degressiven Trend des Phänomens Wohnungseinbruchdiebstähle für den Zeitraum 2015 (167.136) bis 2019 (87.145) sind komplex [2, 3], different interpretierbar und verifizierbar. So spielen die Sensibilisierung der Bevölkerung und damit einhergehende Konsolidierung der Sicherheitstechnik im privaten Umfeld als aktiver und passiver Einbruchsschutz [13, 18], die Strafrechtsänderung aus dem Jahr 2017 mit der Verschärfung der Mindeststrafandrohung von $1 \mathrm{Jahr}$ als Verbrechenstatbestand als negative Generalprävention [4], die Priorisierung dieses Deliktfeldes bei den Polizeien und damit verbundenen repressiven und präventiven Ansätze und nicht zuletzt die Corona-Pandemie mit den restriktiven Quarantänemaßnahmen, z.B. immense häusliche Präsenz durch Homeoffice, sicherlich eine große Rolle. Entsprechend wird sich der degressive Trend der Fallzahlen für dieses Phänomen 2020 fortsetzen [6]. Aus diesem Grund wäre eine Interpretation der Fallzahlen als Beweis für die effektive Wirksamkeit der Prognosemodelle nicht legitim. Die bisherigen durchgeführten, z.T. den Entwicklungsprozess begleitenden Projekt- und Evaluationsstudien reflektieren mit ihren Ergebnissen insbesondere im urbanen Raum moderate bis z.T. hohe Effekte, hingegen im ländlichen Raum geringe bzw. keine Effekte [10, S. 16, 14]. Inwieweit diese Effekte unmittelbar die effektive Wirksamkeit des Prognosemodells und letztlich die Reduzierung des Kriminalitätsaufkommens basierend auf den Ergebnissen des Prognosemodells reflektiert, konnte in unabhängigen empirischen Studien im deutschsprachigen Raum nur teilweise verifiziert werden [9, S. $262 \mathrm{ff}$.$] .$

Bei der Diskussion um die Wirksamkeit der Prognosemodelle insbesondere im internationalen Vergleich sollte nicht außer Acht gelassen werden, dass in Deutschland das Prognostizieren prädiktiver Ereignisse mittels mathematischer Algorithmen im Kontext zur praktischen Polizeiarbeit an die Einhaltung der datenschutzrechtlichen Be- 
stimmungen gebunden ist und somit originär raumbezogene Verfahren Anwendung finden (Ausnahmen angeführt). Aus diesem Grund wäre eine konsequentere, aber auch adäquatere Parametrisierung von für den Algorithmus relevanter Parameter, d.h. stärkere Reflexion auf die Implementierung infrastruktureller und sozioökonomischer Informationen als Umweltindikatoren, z. B. Demografie, Gebäudestruktur, Verkehrsanbindung, Kaufkraftverhalten, Mobilitätsverhalten, Einbruchsaufkommen, Einkommensverteilung, Wetterdaten, Einkaufs- und Freizeitinfrastruktur zielführend [11, S. 18f.]. Dabei scheint der multifaktorielle Ansatz, wie die Risk-Terrain-Methode als nicht originär falldatenorientierte Analysemethode, wie im Bundesland Nordrhein-Westfalen bereits umgesetzt und künftig auch im Bundesland Bayern favorisiert [11, S. 28, 34], im deutschsprachigen Raum erfolgversprechender zu sein. Auch modifizierte falldatenorientierte Near-Repeat-Ansätze werden durch die Assoziation mit weiterführenden allgemeinen Informationen bzw. Daten, d.h. neben aktuellen Ereignissen, allgemein zugänglichen Daten sowie perspektivisch auch Sensordaten, hinsichtlich ihrer prädiktiven Prognose auch optimiert werden. Letztlich dürfte eine Kombination aus verschiedenen Ansätzen und einer Vielzahl an internen und externen Parametern die Prognosewirksamkeit positiver gestalten.

Letztlich hängt der Erfolg des prädiktiven Ansatzes immens von der Determiniertheit und Präzision der sich an die Prognose anschließenden repressiven oder präventiven Exekutivmaßnahmen ab. Aus polizeilicher Sicht definiert sich der Begriff Erfolg insbesondere über konkrete unmittelbare i. S. v. wahrgenommene Parameter, z.B. Festnahmen oder Tatverdächtigenzahl, wogegen die indirekte Form eher zur Inakzeptanz führt. Somit impliziert Wissensmanagement i. S. v. Transferleistung bei Predictive Policing entsprechende Wirkmechanismen, um über den angeregten Kommunikationsprozess Transparenz zu dokumentieren, die letztlich sich in der Motivation der betreffenden Personen widerspiegelt.

Der Prognoseprozess des Predictive Policing ist überwiegend fallzahlenorientiert ausgerichtet. Der Datenerfassungsprozess, v.a. der für die prädiktive Prognose relevanter Daten, wird bei den Polizeien mittels länderspezifischer Vorgangsbearbeitungssysteme i.d.R. manuell im Nachgang, d.h. nach Tatortaufnahme, realisiert und ist hinsichtlich der Datenqualität risikobehaftet. Um die relevante Datenqualität in diesem Datenerfassungsprozess zu gewährleisten, sind Pflichtfelder sowie automatisierte Prüfungen an ausgewählten Prozesspunkten implementiert. Aktuell wird im Prognoseprozess auf ein Phänomen (Wohnungseinbruchdiebstähle) abgestellt und faktisch von übrigen Kriminalitätsaufkommen entkoppelt betrachtet. Aus kriminalistischer Sicht, insbesondere unter Berücksichtigung der mit der prädiktiven Prognose zu erfassenden Zielgruppe (se- mi-/professionelle, planmäßig vorgehende Bande/Gruppe, ggf. mit Bezug zur Organisierten Kriminalität), erscheint diese Vorgehensweise nicht erfolgversprechend. Aus diesem Grund wäre ein Prognosemodell mit einem ganzheitlichen und phänomenübergreifenden Ansatz mit individuellen Optionen für Parameterselektionen, orientiert am Modus Operandi, optimierbar und wahrscheinlich zielführender hinsichtlich der prädiktiven Prognose.

Die im deutschsprachigen Raum veröffentlichten Evaluationsstudien befassen sich in dem Evaluationsprozess originär nur mit der Gruppe der Exekutive. Eine solche Evaluationsstudie kann in erster Linie als Qualitätsmerkmal zur Prüfung der Wirksamkeit interner polizeilicher Prozessabläufe insbesondere in den Bereichen des Wissens-, Qualitätsdaten- und Personalmanagement herangezogen werden. Im Rahmen einer wissenschaftlichen, unabhängigen Evaluationsstudie sollten zur Gewährleistung einer objektiven Reflexion i. S. v. Wirksamkeitsprüfung neben der Gruppe der Exekutive auch Teile der Bevölkerung allgemein, aus pragmatischen Gründen regional begrenzt, und insbesondere die Gruppe der Geschädigten einbezogen werden und zu kriminalgeografischen und -politischen sowie sozioökonomischen und infrastrukturellen Parametern, aber auch zur gefühlten Sicherheit bis hin zur Wahrnehmungsreflexion polizeilicher Arbeit befragt werden; sofern umsetzbar, sollten auch die Gruppe der Täter/-innen in einem solchen Prozess integriert werden, sodass auf prädiktive Prozesse abgestellt werden kann. Nachweislich ändert der unmittelbar betroffene Personenkreis i. S. v. Geschädigten des Phänomens Wohnungseinbruchdiebstähle sein Verhalten insbesondere im Bereich des Sicherheits- und Präventionsverlauf, sodass nachträgliche Maßnahmen zur Sicherung der Wohnung bzw. des Wohnobjektes sich deliktsmindernd auswirken [18, S. 79]; analoges Verhalten ist von Personen zu erwarten, die in unmittelbarer oder mittelbarer Nachbarschaft wohnen, sodass diese Personengruppen prädestiniert für Befragungen wären.

\section{Ausblick}

Das Interesse und die Implementierung mathematischer Algorithmen zur effizienteren und effektiveren Gestaltung der polizeilichen Arbeits- und Entscheidungsprozesse gewinnt immer mehr an Bedeutung. Gerade die Effizienz und Effektivität der Algorithmen hängt dabei im entscheidenden $\mathrm{Ma} ß$ von der Datenqualität ab, die wiederum durch ein funktionierendes Qualitäts- und Wissensmanagementsystem sowie Personalmanagement optimiert werden kann. Tendenziell wird die prädiktive Prognose optional auch mit anderen mathematischen Algorithmen verknüpft werden, z. B. zum Erkennen von analogen Wohnungen bzw. Wohnobjekten aus Luftbildern. Aktuell wird die Qualität der Vorhersagen in 
erster Linie von einem minimalistischen theoretischen Modellansatz getragen [5, S. 14]. Mit fortschreitender Digitalisierung - Big Data \& Internet of Things - werden die quantitativen und qualitativen Anforderungen an polizeiliche Arbeit wachsen. Dabei wird die Erhebung und Verwertung nichtpolizeilicher Daten i. S. v. Umwelt- und Zeitdaten auch in Zukunft für polizeiliche Prognosen immens an Bedeutung zunehmen. Dabei sind ganzheitliche Lösungsansätze, die eine Vielzahl von Algorithmen in den polizeilichen Datenbearbeitungs- und -verarbeitungssystemen implementieren, zu favorisieren. Mit Blick auf die Zukunft der Vorhersage von Kriminalität gerichtet, ist davon auszugehen, dass sich Predictive Policing dauerhaft etablieren und auch stärker ausdifferenzieren lassen wird [11, S. 67].

Funding Open Access funding enabled and organized by Projekt DEAL.

Open Access Dieser Artikel wird unter der Creative Commons Namensnennung 4.0 International Lizenz veröffentlicht, welche die Nutzung, Vervielfältigung, Bearbeitung, Verbreitung und Wiedergabe in jeglichem Medium und Format erlaubt, sofern Sie den/die ursprünglichen Autor(en) und die Quelle ordnungsgemäß nennen, einen Link zur Creative Commons Lizenz beifügen und angeben, ob Änderungen vorgenommen wurden.

Die in diesem Artikel enthaltenen Bilder und sonstiges Drittmaterial unterliegen ebenfalls der genannten Creative Commons Lizenz, sofern sich aus der Abbildungslegende nichts anderes ergibt. Sofern das betreffende Material nicht unter der genannten Creative Commons Lizenz steht und die betreffende Handlung nicht nach gesetzlichen Vorschriften erlaubt ist, ist für die oben aufgeführten Weiterverwendungen des Materials die Einwilligung des jeweiligen Rechteinhabers einzuholen.

Weitere Details zur Lizenz entnehmen Sie bitte der Lizenzinformation auf http://creativecommons.org/licenses/by/4.0/deed.de.

\section{Literatur}

1. Beck C, McCue C (2009) Predictive policing: what can we learn from Wal-Markt and amazon about fighting crime in a rezession? Police Chief 76(11):18-24. https://www.ncjrs.gov/App/ Publications/abstract.aspx?ID=251769. Zugegriffen: 4. Dez. 2020

2. Bundeskriminalamt (2020) Grundtabelle ab 1987 - excel. Polizeiliche Kriminalitätsstatistik. Bundeskriminalamt, Wiesbaden (https://www.bka.de/SharedDocs/Downloads/DE/Publikationen/ PolizeilicheKriminalstatistik/2015/Zeitreihen/Faelle/tb01_Faelle GrundtabelleAb1987_excel.html. Zugegriffen: 4. Dez. 2020

3. Bundeskriminalamt (2020) Tatverdächtige 2019. Polizeiliche Kriminalitätsstatistik Bundesrepublik Deuntschland Jahrbuch 2019, 3, Version 3.0 (67). (Bundeskriminalamt, Hrsg.) Wiesbaden. https:// www.bka.de/DE/AktuelleInformationen/StatistikenLagebilder/ PolizeilicheKriminalstatistik/PKS2019/PKSJahrbuch/pksJahrbuch_ node.html. Zugegriffen: 4. Dez. 2020

4. Bundesrepublik Deutschland (2017) Fünfundfünfzigstes Gesetz zur Änderung des Strafgesetzbuches - Wohnungseinbruchdiebstahl. Bundesgestzblatt Jahrgang 2017 Teil I Nr. 48 (Bundesgestzblatt Jahrgang 2017). (Bundesregierung, Hrsg.) Bonn: Bundesanzeiger Verlag. https://www.bmjv.de/SharedDocs/Gesetzgebungsverfahren/ Dokumente/BGB1_55.AendG_StGB_Wohnungseinbruchdiebstahl. pdf?_blob=publicationFile\&v=3. Zugegriffen: 4. Dez. 2020
5. Demortain D, Benhouzid B (2017) Evaluationg predictive algorithms. In: Leighton A, Bendouzid B, Brice J, Bygrave L, Griffiths A, Lodge M, Yeung A et al (Hrsg) Algorithmic reguation. Discussion paper 85. London School of Econimics and Political Science, London. https://www.kcl.ac.uk/law/research/centres/telos/assets/ DP85-Algorithmic-Regulation-Sep-2017.pdf. Zugegriffen: 4. Dez. 2020

6. Droste J, Brodtmann K, Löwer S (2020) Corona könnte Zahl der Einbrüche auf Allzeittief drücken. Hamburg: Cash.Print GmbH. https://www.cash-online.de/versicherungen/2020/corona-koenntezahl-der-einbrueche-auf-allzeittief-druecken/537972. Zugegriffen: 4. Dez. 2020

7. Earls A (2015) Bessere Datenqualitä beginnt mit besseren Prozessen - nicht mit der Sotfware. ComputerWeekly.de. (T. inc., Hrsg.) Newton. https://www.computerweekly.com/de/feature/BessereDatenqualitaet-beginnt-mit-besseren-Prozessen-nicht-mit-derSoftware. Zugegriffen: 4. Dez. 2020

8. Egbert S (2017) Siegezug der Algorithmen? Predictive Policing im deutschsprachigem Raum. (B. f. Bildung, Hrsg.) Bonn. https:// www.bpb.de/apuz/253603/siegeszug-der-algorithmen-predictivepolicing-im-deutschsprachigen-raum. Zugegriffen: 4. Dez. 2020

9. Egbert S (2018) Predictive Policing in Deutschland. Grundlagen, Risiken, (mögliche) Zukunft. Texte und Ergebnisse des 42. Strafverteidigertages Münster vom 02. bis zum 04.03.2018, S. 241-265. (O. d. Strafverteidigerveeinigungen, Hrsg.) Berlin. https://www.strafverteidigervereinigungen.org/Schriftenreihe/ Texte/Band\%2042/Egbert_241_265_42SchrStVV.pdf. Zugegriffen: 4. Dez. 2020

10. Landeskriminalamt Nordrhein-Westfalen (2018) Abschlussbericht Projekt SKALA - Kurzfassung. (L. Nordrhein-Westfalen, Hrsg.) Düsseldorf. https://lka.polizei.nrw/sites/default/files/201806/180208_Abschlussbericht_SKALA_Kurzfassung.pdf. Zugegriffen: 4. Dez. 2020

11. Krasmann S, Egbert S (2019) Predictive Policing. Eine ethnografische Studie neuer Technologien zur Verhersage von Straftaten und ihre Folgen für die polizeiliche Praxis. Projektabschlussbericht. (U. Hamburg, Hrsg.) Hamburg. https://www.wiso.unihamburg.de/fachbereich-sowi/professuren/hentschel/forschung/ predictive-policing/egbert-krasmann-2019-predictive-policingprojektabschlussbericht.pdf. Zugegriffen: 4. Dez. 2020

12. Montag T (2016) Die Algorithmen des Verbrehcen. Analysen \& Argumente, Ausgabe 215. (Konrad-Adenauer-Stiftung, Hrsg.) Berlin. https://www.kas.de/c/document_library/get_file?uuid=f2a18cbaa6fc-1bc1-9e53-d0b364feab50\&groupId=252038. Zugegriffen: 4 . Dez. 2020

13. Parthier U (2019) Gut oder schlecht? - Datenqualität richtig einschätzen. IT-daily.net. Otterfing: IT Verlag für Informationstechnik GmbH. https://www.it-daily.net/it-management/bigdata-analytics/21214-gut-oder-schlecht-datenqualitaet-richtigeinschaetzen. Zugegriffen: 4. Dez. 2020

14. Shapiro A (2017) Reform predictive policing. Nat Int Wkly J Sci 541(7638):458-460 (https://www.nature.com/news/reformpredictive-policing-1.21338. Zugegriffen: 4. Dez. 2020

15. Sommerer L (2017) Geospatial Predictive Policing-Research Outlook. A Call For Legal Debate. NK Neue Kriminalpolitik. Forum für Kriminalwissenschaften, Recht und Praxis (2), S. 147-164. https://www.nomos-elibrary.de/10.5771/0934-9200-2017-2-147/ geospatial-predictive-policing-research-outlook-a-call-for-legaldebate-jahrgang-29-2017-heft-2. Zugegriffen: 4. Dez. 2020

16. Stelz H, Martin W (2013) Kein Prozess ohne Daten - Datenqualität bestimmt die Prozessqualität. Wissensmanagment Magazin. (B. f. K., Hrsg.) Neusäß: Büro für Medien Oliver Lehnert e. K. https://www.wissensmanagement.net/themen/artikel/ artikel/kein_prozess_ohne_daten_datenqualitaet_bestimmt_die_ prozessqualitaet.html. Zugegriffen: 4. Dez. 2020 
17. Weber M, Gauch S, Amini F, Kaiser T, Tiemann J, Schmidt AO (2015) Vorhersagende Polizeiarbeit. ÖFIT-Trendschau - Öffentliche Informationstechnologie in der digitalisierten Gesellschaft, Trendthema 28. (J. M. Fromm, Hrsg.) Berlin, Berlin, Berlin: Kompetenzzentrum Öffentliche IT. https://www.oeffentliche-it.de/-/ Vorhersagende-Polizeiarbeit. Zugegriffen: 4. Dez. 2020
18. Wollinger G, Dreißigacker A, Blauert K, Bartsch T, Baier D (2014) Wohnungseinbruch: Tat und Folgen. Ergebnisse einer Betroffenenbefragung in fünf Großstädten. Forschungsbericht Nr. 124. (K. F. e. V., Hrsg.) Hannover. https://www.gdv.de/resource/blob/10324/ ce03c269c82143bc97a1cbffdf106990/studie--wohnungseinbruch-tat-und-folgen---1903135228-data.pdf. Zugegriffen: 4. Dez. 2020 